\title{
A Multiplex PCR Assay for the Simultaneous Detection of Chlamydia trachomatis, Neisseria gonorrhoeae, and Trichomonas vaginalis
}

\author{
Ahmad N. Abou Tayoun ${ }^{1}$, Paul R. Burchard ${ }^{1}$, Angela M. Caliendo ${ }^{2}$, Axel Scherer ${ }^{3}$, Gregory \\ J. Tsongalis ${ }^{1}$ \\ ${ }^{1}$ Department of Pathology, Geisel School of Medicine at Dartmouth, Hanover, NH; \\ Dartmouth Hitchcock Medical Center and Norris Cotton Cancer Center, Lebanon, NH, \\ United States \\ ${ }^{2}$ Department of Medicine, Warren Alpert Medical School of Brown University, \\ Providence, RI, United States \\ ${ }^{3}$ Department of Electrical Engineering, California Institute of Technology, Pasadena, CA, \\ United States
}

Send all correspondence to:

Dr. Gregory J. Tsongalis, PhD

Department of Pathology

Dartmouth Hitchcock Medical Center

1 Medical Center Drive

Lebanon, NH 03756

(e): gregory.j.tsongalis@hitchcock.org

(t):603-650-5498 


\begin{abstract}
Introduction: For developing countries, sexually transmitted infections (STIs) and their complications are ranked in the top 5 disease categories for which adults seek medical treatment. Chlamydia trachomatis (CT), Neisseria gonorrhoeae (NG), and Trichomonas vaginalis (TV) are the three most common STIs worldwide, with TV accounting for over half of the cases. In developing countries, traditional methods for diagnosing STIs are laborious, often not very sensitive, and have a long turnaround time with most recent commercially available diagnostic tests targeting one or, at most, two of these STIs at a time. Here, we describe the development of a highly sensitive, rapid and affordable sample-to-answer multiplex PCR-based assay for the simultaneous detection of Trichomonas vaginalis, Neisseria gonorrhoeae, and Chlamydia trachomatis.
\end{abstract}

Materials and Methods: We designed a multiplex PCR assay for the detection of 4 targets (CT, TV, NG, and process/PCR control) using melt curve analysis. To establish the limit of detection (LOD) for each pathogen, we used previously extracted and quantified TV, NG, and CT genomic DNA (Vircell, Spain). For each target, the LOD was determined by lowering its copy number while increasing the other two STI loads in a stepwise fashion. The process/PCR control remained constant in the optimized assay and was spiked into each sample before extraction. For a concordance study, we tested urine, vaginal and rectal swab specimens from 26 patients positive for one or more of the tested STIs. In addition, 56 liquid cytology specimens (Thinprep) were used to assess specificity. 
Results: This assay has a turnaround time of less than 2 hours and has a limit of detection as low as $7-31$ copies for each STI in the presence of the other 2 targets. Our assay also demonstrated $100 \%$ concordance with 26 known clinical samples from urine, vaginal and rectal swab specimens. TV, NG, CT, and our process/PCR control were consistently identified at $78^{\circ} \mathrm{C}, 82.3^{\circ} \mathrm{C}, 85.7^{\circ} \mathrm{C}$, and $\sim 92^{\circ} \mathrm{C}$, respectively. When applied to DNA extracted from residual Thinprep specimens, the assay was negative in 54/56 samples. Two samples were found to be co-infected with CT.

Conclusions: Our multiplex assay combines a rapid and cost-effective approach to molecular diagnostics with the versatility required for use within a variety of laboratory settings. These performance characteristics make this multiplex STI assay highly suitable for use in a clinical laboratory. 


\section{Introduction}

An estimated 499 million new cases of curable sexually transmitted infections (STIs) occur every year, with around 19 million in the United States alone ${ }^{1-4}$. For developing countries, STIs and their complications are ranked in the top 5 disease categories for which adults seek medical treatment ${ }^{2,5}$. Chlamydia trachomatis (CT), Neisseria gonorrhoeae (NG), and Trichomonas vaginalis (TV) are the three most common STIs worldwide, with TV accounting for over half of the cases ${ }^{6-10}$. Such STIs often appear asymptomatic and, if untreated, can have devastating consequences on reproduction, maternal and newborn health, as well as increase the likelihood of acquisition and transmission of HIV ${ }^{6,11-15}$. The World Health Organization (WHO) has identified rapid, low-cost, and accurate point-of-care diagnostic tests as a key point for action in the global strategy for the prevention and control of STIs ${ }^{2,16}$.

In developing countries, traditional methods for diagnosing STIs are laborious, often not very sensitive, and have a long turnaround time with most recent commercially available diagnostic tests targeting one or, at most, two of these STIs at a time ${ }^{6,9,17-19}$. However, studies have shown that $45.7 \%$ of persons infected with NG, are co-infected with $\mathrm{CT}^{20}$. With approximately 106 million new Neisseria gonorrhoeae cases occurring every year, such co-infections are abundant in the population ${ }^{2}$. In addition, the incidence of TV co-infection with either CT, NG or both accounts for over $2 \%$ of STI cases or roughly 11 million cases per year ${ }^{21,22}$. This combined with the high prevalence of TV 
signifies the necessity for testing all three targets in any given-preferably easy to obtainspecimen thereby preserving samples, lowering cost, enhancing clinical sensitivity, and reducing turnaround time for more efficient treatment.

PCR has been the cornerstone of molecular diagnostics in clinical laboratories. This testing method combines high levels of sensitivity, specificity, and accuracy, with rapid turnaround times, making for a more appealing alternative to culture-based testing methods which are often limited by long culture times and low sensitivity with certain sample types ${ }^{23-26}$. PCR-based assays, on the other hand, are compatible with several sample types that can be used directly for DNA extraction without the need for culture ${ }^{27}$.

Here, we describe the development of a highly sensitive, rapid and affordable multiplex PCR-based assay for the simultaneous detection of Trichomonas vaginalis, Neisseria gonorrhoeae, and Chlamydia trachomatis.

\section{Materials and Methods}

\section{Samples}

A total of 83 patient samples were used in this study. In the concordance study, 26 samples including 10 urine, 9 vaginal and 7 rectal swab specimens were obtained from patients that have previously tested positive for CT and NG using the Abbott m2000 
real-time CT/NG assay, and TV using a laboratory developed Real-Time PCR assay ${ }^{28}$. For the sensitivity study, limit of detection was established using genomic DNA extracted from known numbers of TV, NG, and CT organisms (Vircell, Spain). For the specificity study, we used 57 female patient samples previously tested for Human Papillomavirus (HPV) with the Roche $\mathrm{COBAS}^{\circledR} 4800$ HPV Assay.

\section{Extraction}

For each patient sample, $2 \mathrm{ml}$ (urine) or $600 \mu \mathrm{l}$ buffer (swabs) was pelleted by centrifugation at $6000 \mathrm{rpm}$ for 10 minutes and re-suspended with $195 \mu$ l of water. Each sample was then spiked with $5 \mu \mathrm{l}$ of $2.5 \mathrm{pg} / \mu \mathrm{l}$ internal control DNA $\left(\mathrm{IC}_{2} \mathrm{M}\right.$, see table 1$)$. DNA was then extracted with the Qiagen EZ1 robotic system using the bacterial DNA card and the EZ1 tissue extraction kit/cartridges (Qiagen, Valencia, CA). DNA was eluted in $50 \mu$ l elution buffer, of which $10 \mu$ l were used for the $25 \mu$ PCR reactions.

\section{PCR}

Internal control $\left(\mathrm{IC}_{2} \mathrm{M}\right)$ and primer sequences (Table 1) were synthesized by Integrated DNA Technologies (Coralville, lowa). The primer sequences were compared in silico to human, viral, and bacterial genomes within the NCBI database. No significant similarities between the targeted sequences and any of the tested genomes that could theoretically lead to non-specific amplification were found. 
For patient samples, we prepared $25 \mu$ I PCR reactions consisting of $1 \mathrm{X} \mathrm{SsoFast}^{\mathrm{TM}}$ EvaGreen ${ }^{\circledR}$ Supermix, $10 \mu$ l extracted DNA (this should theoretically contain $\sim 2.5 \mathrm{pg}$ $\mathrm{IC}_{2} \mathrm{M}$, see above), $100 \mathrm{nM}$ forward and reverse $\mathrm{CT}$ primers, $175 \mathrm{nM}$ forward and reverse NG primers, $125 \mathrm{nM}$ forward and reverse TV primers and $150 \mathrm{nM} \mathrm{IC} \mathrm{C}_{2} \mathrm{M}$ forward and reverse primers. For the limit of detection studies, DNA extracted from a known copy number of each target was spiked into the PCR reaction, in addition to $2.5 \mathrm{pg} I \mathrm{IC}_{2} \mathrm{M}$.

PCR amplification was performed on the SmartCycler ${ }^{\circledR}$ (Cepheid, Sunnyvale, CA) using a touchdown PCR protocol (Figure $1 \mathrm{~A})$ that includes a 5 minute hot start $\left(95^{\circ} \mathrm{C}\right)$ followed by two cycles of 5 second denaturation $\left(95^{\circ} \mathrm{C}\right)$ and 30 second annealing/extension at $70^{\circ} \mathrm{C}$, two cycles of 5 at $95^{\circ} \mathrm{C}$ and 30 second at $67^{\circ} \mathrm{C}$, two cycles of 5 second at $95^{\circ} \mathrm{C}$ and 30 second at $65^{\circ} \mathrm{C}$, two cycles of 5 second at $95^{\circ} \mathrm{C}$ and 30 second at $63^{\circ} \mathrm{C}$, and finally 32 cycles of 5 second at $95^{\circ} \mathrm{C}$ and 30 second at $60^{\circ} \mathrm{C}$. A melt protocol was also included from $60-95^{\circ} \mathrm{C}$ at $0.2^{\circ} \mathrm{C} /$ second (Figure $1 \mathrm{~A}$ ).

\section{Results}

\section{Multiplex STI assay}

Our PCR-based assay was developed for the simultaneous detection of three major STIS (CT, NG, and TV) in addition to a novel internal control DNA sequence (IC $\left.C_{2} M\right)$. The latter was spiked into each patient sample before extraction and thus served as both a process 
and PCR control (see materials and methods). All primer sequences were carefully designed and selected to clearly resolve the characteristic intercalating dye-based melt peaks for the four different targets in our multiplex assay (Figure 1B). In our primer design, we targeted multi-copy regions within the three STI genomes to achieve highest sensitivity. For Chlamydia trachomatis, we targeted the multi-copy cryptic plasmid thus, not only enhancing sensitivity, but also ensuring the detection of all Chlamydia trachomatis serovars including the Swedish nvCT serovar ${ }^{28,29}$. After extensive PCR optimization, we devised a touchdown PCR protocol with extension temperatures gradually decreasing from $70^{\circ} \mathrm{C}$ to $60^{\circ} \mathrm{C}$ as shown in Figure $1 \mathrm{~A}$. With this optimal protocol, we were able to precisely resolve the melt peaks for TV at $78^{\circ} \mathrm{C}, \mathrm{NG}$ at $82.3^{\circ} \mathrm{C}$, $\mathrm{CT}$ at $85.7^{\circ} \mathrm{C}$, and $\mathrm{IC}_{2} \mathrm{M}$ at $92^{\circ} \mathrm{C}$ (Figure $1 \mathrm{~B}$ ).

Sensitivity or Limit of detection

To establish the limit of detection (LOD) for each pathogen, we spiked into our PCR reactions genomic DNA extracted from known copy numbers of TV, NG, and CT (Vircell, Spain). For each target, the LOD was determined by lowering its copy number while increasing the other two STI loads in a stepwise fashion (Tables 2-4). For example, the LOD for Neisseria gonorrhoeae was 7 or 15 copies/reaction even when either TV or CT was present at a high concentration (3000 copies/reaction), respectively. This LOD was increased to 31 copies/reaction when both TV and CT were each present at 3000 copies/reaction (Table 2 and Figure 2). Although the LOD for either TV or CT was 7 copies/reaction in the absence of the other two pathogenic targets, this LOD ranged 
between 7 to 31 copies/reaction as the other STIs were increasingly spiked (up to 3000 copies) into the PCR reaction (Tables 3 and 4). Overall, our assay demonstrates a very high sensitivity for the detection of each of the STI targets even when the other targets were present at very high copy numbers.

\section{Specificity}

We assessed the specificity of the assay by testing specimens which had been previously submitted for HPV screening. We extracted DNA from 56 female patient ThinPrep liquid cytology specimens. Twelve of these specimens were positive for high risk HPV. Of the 12 positive HPV samples, we identified 2 as also positive for CT. This was not a surprising finding as co-infection of HPV and CT occurs in approximately $14 \%$ of HPV positive women $^{31}$, which is consistent with our findings. The other HPV positive samples as well as the 44 HPV negative samples showed only amplification of the internal control. This suggests proper DNA extraction and no cross reactivity between positive HPV samples or genomic DNA with our assay. In addition, we tested five gram-positive and five gramnegative bacteria samples representing normal vaginal flora. Our assay identified all ten of these bacteria samples as negative for our STI targets, which was expected. This suggests no cross reactivity between our assay and common vaginal flora.

Furthermore, of the 26 known positive swab and urine samples (see below), our assay never gave an unexpected melt peak for any of the tested targets. This further supports 
our primer sets as specific for each target and that they do not cross react with one another or the internal control, even at high concentrations.

\section{Accuracy}

To determine the accuracy of our assay, we extracted DNA from 26 samples for patients previously shown to be positive for $\mathrm{CT}, \mathrm{NG}$, and/or TV and tested them using our assay. The specimens used for this accuracy study were from urine, vaginal swabs, and rectal swabs. Our assay correctly identified 26 of the 26 previously tested patient samples. TV and CT infections were found in 8 samples and NG infection was found in 6 samples. Coinfections with CT and TV, NG and TV, and CT and NG, were found in 1 sample each. These results were $100 \%$ concordant with what was expected and demonstrate a high level of accuracy for our assay from a variety of specimen sources.

\section{Turnaround Time}

The total turnaround time for this assay is less than 2 hours. The assay begins with a 10 minute centrifugation, followed by a 15 minute automated extraction. PCR amplification takes approximately 1 hour to set up and run. The results of the melt-curve are available within a few minutes of completion of the amplification and easy to interpret. 


\section{Discussion}

In this study, we report proof of principle for a new multiplex method for the simultaneous detection of the three most common STIs; TV, CT, and NG from various specimen sources including Thinprep liquid cytology samples. This multiplex PCR-based assay uses an intercalating dye and melt-curve analysis to determine the presence of each STI. Such an assay can be made affordable, highly sensitive and can be performed on real-time PCR platforms common to any molecular diagnostics laboratory. Multiplexing provides the advantage of detecting multiple targets at the same time and from the same specimen. Furthermore, the ability to limit the diagnostic process to one test minimizes both cost and time.

Unlike fluorescently labeled probes, the use of intercalating dyes and melt curve analysis greatly lowers the cost of this assay while maintaining high specificity and sensitivity. Our assay also uses an automated DNA extraction system, which minimizes the required hands-on time. This not only limits the possibility of contamination but also makes for a safer extraction process. The turnaround time for our assay is less than 2 hours, which drastically reduces the turnaround time as compared to more traditional culture based methods.

Our results demonstrate this assay as highly sensitive, specific, and accurate. The LOD for this assay was very low for each STI, even in the presence of high copy numbers of 
one or both of the other STIs. Our assay will therefore be able to easily identify coinfection amongst all three of these STIs. In addition, our results were $100 \%$ concordant with what was expected for the previously tested patient specimens. Specimens containing one or more of the desired STIs were equally identifiable, which was expected. In addition, these specimens exhibit the wide range of samples that can be used in our assay, such as urine, vaginal swabs, and cervical cytology specimens.

This assay combines a rapid and cost-effective approach to molecular diagnostics with the versatility required for use within a variety of laboratory settings. These performance characteristics make this multiplex STI assay highly suitable for use in a clinical laboratory.

\section{ACKNOWLEDEGMENTS}

The authors would like to thank Cheryl Bissaillon from the Baystate Health Center for generously providing control samples. The authors wish to thank the staff of the DHMC Molecular Pathology Laboratory and the Translational Research Program. The data presented in this manuscript was in part generated through the Department of Pathology Translational Research Shared Resource Laboratory of the Geisel School of Medicine at Dartmouth, the Dartmouth Hitchcock Medical Center and the Norris Cotton Cancer Center. This work was supported by the Bill and Melinda gates Foundation [Global Health Grant Number OPP1028794].

\section{References:}


1. http://wwwnc.cdc.gov/travel/yellowbook/2012/chapter-3-infectious-diseasesrelated-to-travel/sexually-transmitted-diseases (Last date accessed: 10/5/13)

2. http://www.who.int/mediacentre/factsheets/fs110/en/index.html (Last date accessed: 10/8/13)

3. Owusu-Edusei K Jr, Chesson HW, Gift TL, et al. The estimated direct medical cost of selected sexually transmitted infections in the United States, 2008. Sexually Transmitted Diseases. 2013;40(3):197-201.

4. Chesson HW, Blandford JM, Gift TL, et al. The estimated direct medical cost of sexually transmitted diseases among American youth, 2000. Perspectives on Sexual and Reproductive Health. 2004;36(1):11-9.

5. Diclemente RJ, Wingood GM, Crosby RA, et al. A descriptive analysis of STD prevalence among urban pregnant African American teens: data from a pilot study. The Journal of Adolescent Health. 2004;34(5):376-83.

6. http://www.cdc.gov/std/stats10/default.html (Last date accessed: 10/15/13)

7. Manam S, Chaganty BK, Evani SJ, et al. Intranasal vaccination with Chlamydia pneumonia induces cross-species immunity against genital Chlamydia muridarum challenge in mice. PLoS One. 2013;8(5):e64917.

8. Coleman JS, Gaydos CA, Witter F. Trichomonas vaginalis in obstetrics and gynecology practice: new concepts and controversies. Obstetrical \& Gynecological Survey. 2013;68(1):43-50.

9. Mahony JB, Luinstra KE, Tyndall M, et al. Multiplex PCR for detection of Chlamydia trachomatis and Neisseria gonorrhoeae in Genitourinary specimens. Journal of Clinical Microbiology. 1995;33(11):3049.

10. Blake DR, Maldeis N, Barnes MR, et al. Cost-effectiveness of screening strategies for Chlamydia trachomatis using cervical swabs, urine, and self-obtained vaginal swabs in a sexually transmitted disease clinic setting. Sexually Transmitted Diseases. 2008;35(7):649-55.

11. Katusiime C, Schlech WF ${ }^{\text {rd }}$, Parkes-Ratanshi R, et al. Characteristics of Sexually Transmitted Infections among High-Risk HIV-Positive Patients Attending an Urban Clinic in Uganda. Journal of the International Association of Providers of AIDS Care. 2013. 
12. Gottlieb S, Newman L, Amin A, et al. Sexually transmitted infections and women's sexual and reproductive health. International Journal of Gynecology \& Obstetrics. 2013.

13. Theunissen KA, Hoebe $\mathrm{CJ}$, Crutzen $\mathrm{R}$, et al. Using intervention mapping for the development of a targeted secure web-based outreach strategy named SafeFriend, for Chlamydia trachomatis testing in young people at risk. BMC Public Health. 2013;13(1):996.

14. Al-Moushaly A. Considerations on male infertility in genital infections with Chlamydia Trachomatis (CT). Journal of Medicine and Life. 2013;6(3):283-6.

15. Beharry MS, Shafii T, Burstein GR. Diagnosis and treatment of chlamydia, gonorrhea, and trichomonas in adolescents. Pediatric Annals. 2013;42(2):26-33.

16. Hsieh YH, Gaydos CA, Hogan MT, et al. What qualities are most important to making a point of care test desirable for clinicians and others offering sexually transmitted infection testing? PLoS One. 2011;6(4):e19263.

17. Marangoni A, Foschi $C$, Nardini $P$, et al. Evaluation of the new test VERSANT CT/GC DNA 1.0 assay for the detection of Chlamydia trachomatis and Neisseria gonorrhoeae in urine specimens. Journal of Clinical Laboratory Analysis. 2012;26(2):70-2.

18. Doseeva V, Forbes T, Wolff J, et al. Multiplex isothermal helicase-dependent amplification assay for detection of Chlamydia trachomatis and Neisseria gonorrhoeae. Diagnostic Microbiology and Infectious Disease. 2011;71(4):354-65.

19. Choe HS, Lee DS, Lee SJ, et al. Performance of Anyplex ${ }^{\mathrm{TM}}$ II multiplex real-time PCR for the diagnosis of seven sexually transmitted infections: comparison with currently available methods. International Journal of Infectious Diseases. 2013.

20. Datta S, Sternberg M, Johnson R, et al. Gonorrhea and Chlamydia in the United States among Persons 14 to 39 Years of Age, 1999 to 2002. Annals of Internal Medicine. 2007;147(2):89-96.

21. Ginocchio CC, Chapin K, Smith JS, et al. Prevalence of Trichomonas vaginalis and Coinfection with Chlamydia trachomatis and Neisseria gonorrhoeae in the United States as Determined by the Aptima Trichomonas vaginalis Nucleic Acid Amplification Assay. Journal of Clinical Microbiology. 2012;50(8):2601-2608. 
22. van Veen MG, Koedijk FD, van der Sande MA. STD coinfections in The Netherlands: Specific sexual networks at highest risk. Sexually Transmitted Diseases. 2010;37(7):416-22.

23. Espy MJ, Uhl JR, Sloan M, et al. Real-Time PCR in Clinical Microbiology: Applications for Routine Laboratory Testing. Clinical Microbiology Reviews. 2006;19(1):165-256.

24. Palavecino EL. Rapid methods for detection of MRSA in clinical specimens. Methods in Molecular Biology. 2014;1085:71-83.

25. Braverman PK, Schwarz DF, Mph M, et al. Use of ligase chain reaction for laboratory identification of Chlamydia trachomatis and Neisseria gonorrhoeae in adolescent women. Journal of Pediatric and Adolescent Gynecology. 2002;15(1):37-41.

26. Battle TJ, Golden MR, Suchland KL, et al. Evaluation of laboratory testing methods for Chlamydia trachomatis infection in the era of nucleic acid amplification. Journal of Clinical Microbiology. 2001;39(8):2924-7.

27. Kilmarx PH, Black CM, Limpakarnjanarat $\mathrm{K}$, et al. Rapid assessment of sexually transmitted diseases in a sentinel population in Thailand: prevalence of chlamydial infection, gonorrhoea, and syphilis among pregnant women-1996. Sexually Transmitted Infections. 1998;74(3):189-93.

28. Caliendo AM, Jordan JA, Green, AM, Ingersoll J, Wingood GM, and DiClemente RJ. Real-Time PCR Provides Improved Detection of Trichomonas vaginalis Infection Compared to Culture Using Self-Collected Vaginal Swabs. Infectious Diseases in Obstetrics and Gynecology. 2005;13:145-150.

29. Unemo M, Clarke IN. The Swedish new variant of Chlamydia trachomatis. Current Opinion in Infectious Diseases. 2011;24(1):62-69.

30. Palmer L, Falkow S. A common plasmid of Chlamydia trachomatis. Plasmid. 1986;16(1):52-62.

31. Verteramo R, Pierangeli A, Mancini E, et al. Human Papillomaviruses and genital coinfections in gynaecological outpatients. BMC Infectious Diseases. 2009;9(16).

32. Trichomonas vaginalis detection using real-time TaqMan PCR. Journal of Microbiological Methods. 2007;68(2):243-247.

33. A Fast Real-Time Polymerase Chain Reaction Method for Sensitive and Specific Detection of the Neisseria gonorrhoeae porA Pseudogene. Journal of Molecular Diagnostics. 2006;(8)5:574-581. 
Figure Legends

Figure 1. A Representation of touchdown real-time PCR reaction profile. B SmartCycler real-time PCR melt curve analysis results for Neisseria gonorrhoeae (NG), Chlamydia trachomatis (CT), and Trichomonas vaginalis (TV) with equal copy numbers of each in the reaction mixture.

Figure 2. SmartCycler real-time PCR melt curve analysis results for Neisseria gonorrhoeae (NG) limit of detection. Plotted as the fluorescence with respect to temperature. Copy number refers to the copies of NG in the reaction mixture. High Chlamydia trachomatis (CT) and Trichomonas vaginalis (TV) corresponds to 3000 copies of each in the reaction mixture. 
Table 1: Sequences

\begin{tabular}{|c|c|c|c|}
\hline Oligo name & Sequence & Target & Source \\
\hline $\begin{array}{l}\text { Internal Control } \\
\left(\mathrm{IC}_{2} \mathrm{M}\right)\end{array}$ & $\begin{array}{l}\text { TTGTATACCGTGAGCACCACAATTCCCCAGGG } \\
\text { TACTATGACGGTCGTTACTGGACAATGTGGCA } \\
\text { GTTGCCCAGGTTCGGGCGCGCTGCCCCGGCCC } \\
\text { CGGCTCTGAGTGGGCCCGACGACTGCGAGGA } \\
\text { GGCGCACCCCGACGCCTTCATCCGTATCATTG } \\
\text { GATTCGACAGCAACCGTCAAGTCCAATGTGTC } \\
\text { AGTTTCATT }\end{array}$ & NA & $\begin{array}{l}\text { Modified from } \\
\text { Spinacia } \\
\text { oleracea } \\
\text { chloroplast } \\
\text { ribulose 1,5- } \\
\text { bisphosphate } \\
\text { carboxylase } \\
\text { small subunit } \\
\text { gene (GenBank: } \\
\text { L776557.1) }\end{array}$ \\
\hline $\begin{array}{l}\mathrm{IC}_{2} \mathrm{M} \text { Forward } \\
\mathrm{IC}_{2} \mathrm{M} \text { Reverse }\end{array}$ & $\begin{array}{l}\text { 5'-GGTCGTTACTGGACAATGT-3' } \\
\text { 5'-GAATCCAATGATACGGATGAA-3' }\end{array}$ & IC2M & In-house \\
\hline $\begin{array}{l}\text { CT Forward } \\
\text { CT Reverse }\end{array}$ & $\begin{array}{l}\text { 5'-TCTGAGCACCCTAGGCGTTT -3' } \\
\text { 5'-CGTAACTCGCTCCGGAAAAA-3' }\end{array}$ & Cryptic plasmid & In-house \\
\hline $\begin{array}{l}\text { NG Forward } \\
\text { NG Reverse }\end{array}$ & $\begin{array}{l}\text { 5'-CCGGAACTGGTTTCATCTGATT-3' } \\
\text { 5'-GTTTCAGCGGCAGCATTCA-3' }\end{array}$ & porA psuedogene & Reference 32 \\
\hline $\begin{array}{l}\text { TV Forward } \\
\text { TV Reverse }\end{array}$ & $\begin{array}{l}\text { 5'-AAGATGGGTGTTTTAAAGCTAGATAAGGT-3' } \\
\text { 5'-CGTCTTCAAGTATGCCCCAGTAC-3' }\end{array}$ & 2-kb repeated target & Reference 33 \\
\hline
\end{tabular}


Table 2: Chlamydia trachomatis Limit of detection

\begin{tabular}{ccc}
\hline \hline $\begin{array}{c}\text { Chlamydia trachomatis } \\
\text { (LOD) }\end{array}$ & Neisseria gonorrhoeae & Trichomonas vaginalis \\
\hline 7 & 0 & 0 \\
7 & Low* & Low \\
15 & High** & 0 \\
15 & 0 & High \\
31 & High & High \\
\hline
\end{tabular}

*7 copies per reaction

**3000 copies per reaction 
Table 3: Neisseria gonorrhoeae Limit of detection

\begin{tabular}{ccc}
\hline \hline $\begin{array}{c}\text { Neisseria gonorrhoeae } \\
(\text { LOD })\end{array}$ & Chlamydia trachomatis & Trichomonas vaginalis \\
\hline 7 & & 0 \\
7 & 0 & Low \\
15 & Low* & 0 \\
7 & High** & High \\
31 & 0 & High \\
\hline
\end{tabular}

*7 copies per reaction

$* * 3000$ copies per reaction 
Table 4: Trichomonas vaginalis Limit of detection

\begin{tabular}{ccc}
\hline \hline $\begin{array}{c}\text { Trichomonas vaginalis } \\
(\text { LOD })\end{array}$ & Chlamydia trachomatis & Neisseria gonorrhoeae \\
\hline 7 & & 0 \\
7 & Low* & Low \\
15 & High** & 0 \\
15 & 0 & High \\
15 & High & High \\
\hline
\end{tabular}

*7 copies per reaction

**3000 copies per reaction 


\section{Figure 1: Touchdown PCR and Melt Curve Analysis}

A
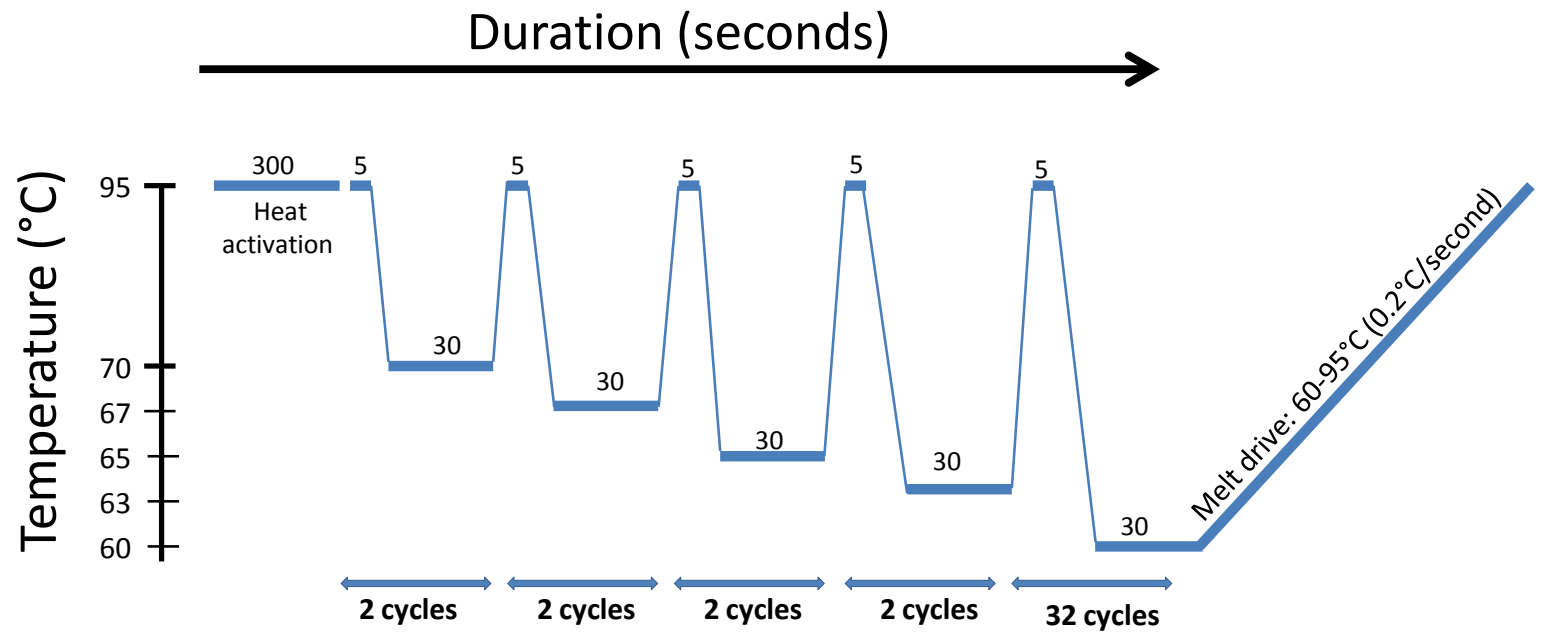

B

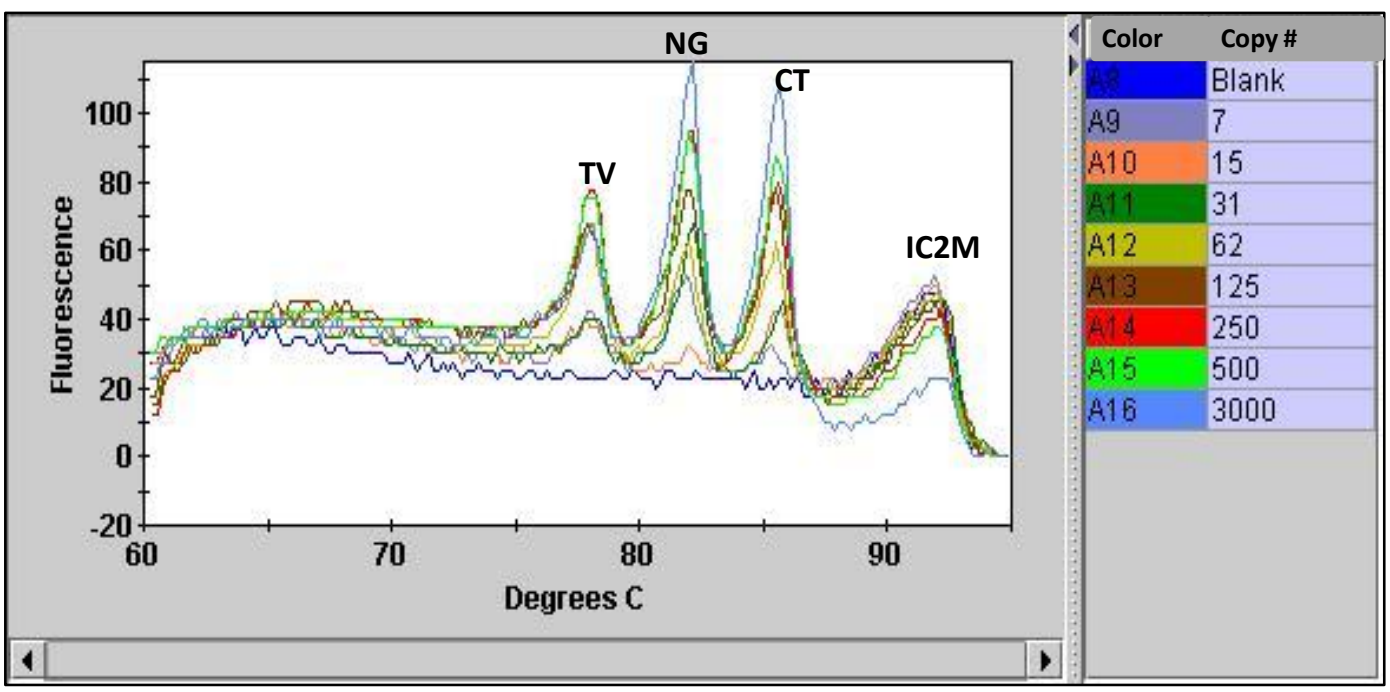


Figure 2: Neisseria gonorrhoeae limit of Detection

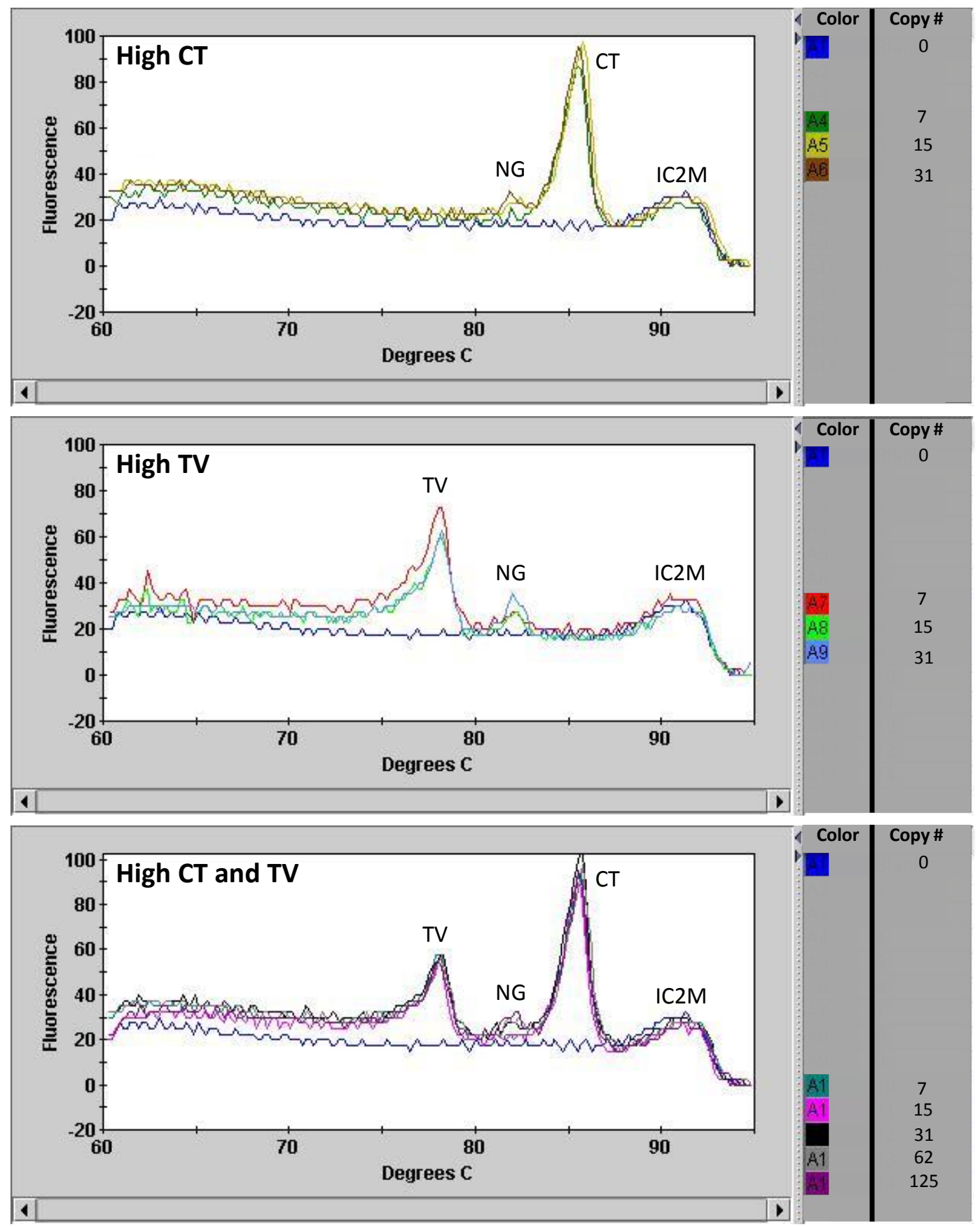

